\title{
Abklärung bei Frauen nach der Menopause - Werden die Leitlinien berücksichtigt?
}

\author{
Die aktualisierten Leitlinien der US-amerikanischen urologi- \\ schen Fachgesellschaft empfehlen grundsätzlich schon bei ein- \\ maligem Nachweis einer asymptomatischen Mikrohämaturie \\ (AMH) ab dem 35. Lebensjahr eine Zystoskopie und Mehrpha- \\ sen-Kontrastmittel-CT von Niere und ableitenden Harnwegen, \\ um ein Malignom auszuschließen. Medizinerinnen der Duke \\ University haben die Anwendung der Leitlinien bei postmeno- \\ pausalen Frauen untersucht. \\ J Urol 2016; 195: 937-941
}

\section{mit Kommentar}

Bei mehr als einem Viertel aller Frauen, die wegen einer nach der Menopause auftretenden AMH untersucht werden, geschieht das nicht in Übereinstimmung mit den Leitlinien der American Urological Association. $\mathrm{Zu}$ diesem Ergebnis komme Megan Bradley und ihre Kolleginnen, die Daten von 237 zwischen August 2012 und August 2014 in ihrem Zentrum untersuchten Frauen in ihre retrospektive Auswertung aufgenommen haben.
Eingeschlossen wurden alle Frauen ab dem 55. Lebensjahr (im Durchschnitt 67 Jahre). Bei ihnen überprüften die Wissenschaftlerinnen zunächst das Vorliegen einer „echten“ AMH, also den mikroskopischen Nachweis von $\geq 3$ Erythrozyten pro Gesichtsfeld bei größtmöglicher Auflösung. Frauen mit einer geringeren Erythrozytenzahl oder solche, deren AMH-Diagnose lediglich auf einen für Blut positiven Urin-Stix zurückging, wurden als

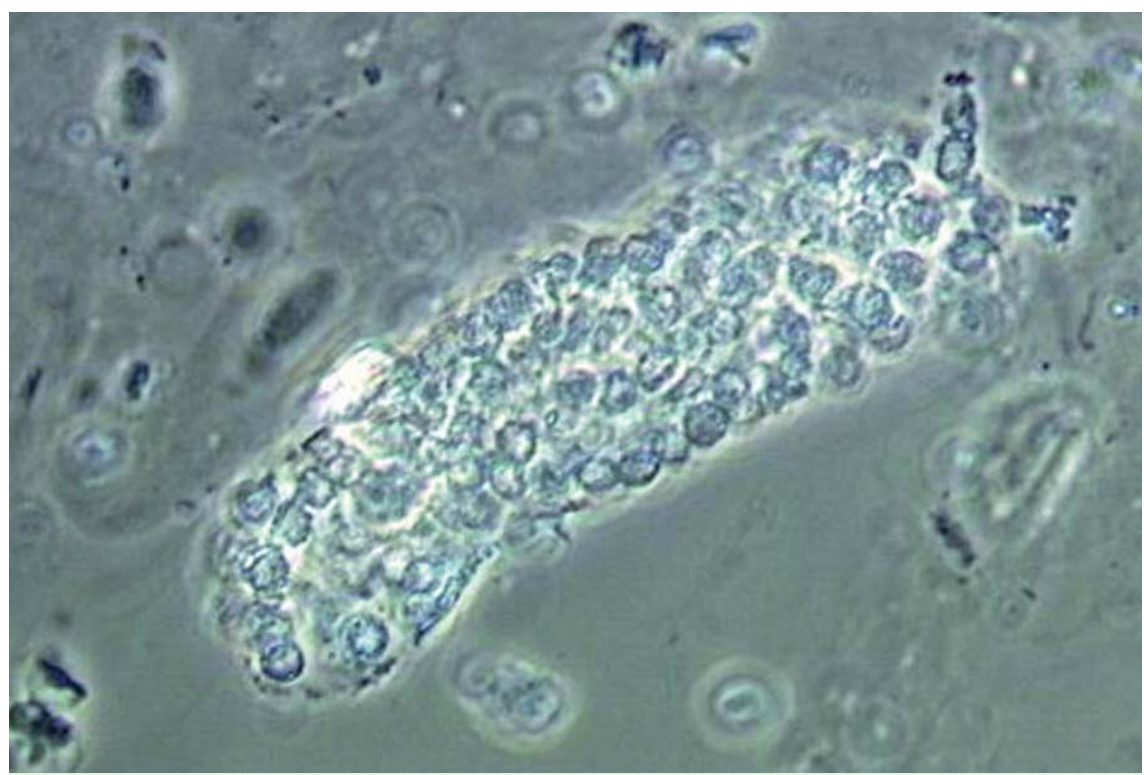

Erythrozytenzylinder im Urin sind beweisend für eine glomeruläre / renale Ursache der Hämaturie (phasenkontrastmikroskopisches Bild). (Bild: Beetz R, Walz PH. Differenzialdiagnose der Hämaturie. In: Stein R, Beetz R, Thüroff JW, Hrsg. Kinderurologie in Klinik und Praxis. 3. Aufl. Stuttgart: Thieme; 2011)
„Stix-positiv“ betrachtet. Anschließend wurden aus den Akten das weitere Vorgehen der behandelnden Ärzte und die endgültigen Diagnosen entkommen.

Dabei fand sich bei knapp drei Viertel der Frauen eine echte AMH nach obiger Definition ( $n=169 ; 71,3 \%)$. 40 Frauen wiesen in der Urinuntersuchung weniger als 3 Erythrozyten pro Gesichtsfeld auf, und bei 48 Frauen lag lediglich ein positiver StixTest vor. Nur bei 60 Frauen wurde bereits am Tag des positiven Urin-Stix-Tests eine Urinprobe entnommen und eingeschickt, die restlichen Patientinnen wurden zur Abklärung der AMH überwiesen, ohne dass diese Diagnose gesichert war. Insgesamt 234 Frauen wurden zystoskopiert.

Nur bei 150 Frauen erfolgte eine vollständige gynäkologische Untersuchung zur Abklärung einer Atrophie des Vaginalepithels und eines urogenitalen Prolaps, die ebenfalls Ursachen einer AMH sein können. Eine vaginale Atrophie lag bei 90 Frauen vor, ein Prolaps Stadium II oder höher bei 17 Frauen. Bei 36 Frauen bestand der Verdacht auf einen rezidivierenden Harnwegsinfekt.

Insgesamt fand sich bei 3 der 210 Frauen $(1,4 \%)$, die vollständig untersucht worden waren, ein Malignom des Harntrakts: 1 Nierenkarzinom bei einer Stix-positiven Frau (Testreifen 1+ auf Blut) und 2 Blasenkarzinome bei Frauen mit echter AMH.

\section{Fazit}

Bei $28,7 \%$ der hier beschriebenen Frauen, bei denen mit teilweise aufwendigen Methoden die Ursache einer AMH geklärt werden sollte, lag per definitionem überhaupt keine AMH vor. Darüber hinaus wurden Patientinnen oft schon zur weiteren Diagnostik überwiesen, bevor überhaupt die Sicherung der AMH versucht wurde. Hätte zunächst eine mikroskopische Untersuchung des Urinsediments stattgefunden bzw. wäre eine Urinkultur angelegt worden, hätte eine erhebliche Zahl von Untersuchungen vermieden werden können. Dabei war die Häufigkeit von Malignomen in dieser Gruppe der postmenopausalen Frauen gering, sodass die Leitlinien bei ihren diagnostischen Empfehlungen weitere Faktoren berücksichtigen sollten, bevor eine Zystoskopie gefordert wird, so die Autoren.

\section{Dr. Elke Ruchalla, Bad Dürrheim}


Mikrohämaturie: „Makrohämaturie light" - oder noch weniger?

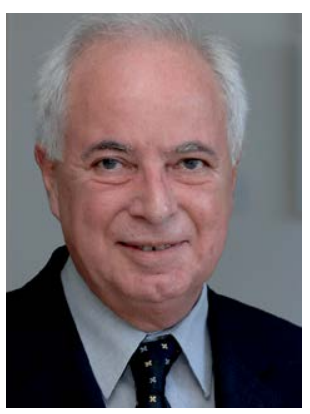

Prof. Dr. Helmut Haas ist niedergelassener Urologe in Heppenheim an der Bergstraße

und Honorarprofessor an der Urologischen Klinik der Johannes Gutenberg-Universität Mainz

Wir alle haben mit der medizinischen Muttermilch eingesogen, dass die Abklärung einer asymptomatischen Mikrohämaturie ein wichtiges Instrument ist, um urologische Malignome zu entdecken. Aus unserer Tätigkeit in der Klinik sprechen dafür zwei Beobachtungen: Bei einer Makrohämaturie finden wir häufig einen Tumor, und der weitaus überwiegende Teil unserer Blasentumorpatienten hat zum mindesten eine Mikrohämaturie.

\section{Überraschende Zahlen?}

Umso ernüchternder sind die Daten aus der Literatur: In einer sehr groß angelegten Studie der Arbeitsgruppe um Jung [1] wurden bei 156691 Probanden nur bei $0,68 \%$ urologische Tumoren gefunden, in der um Loo [2] bei 2630 Probanden bei $1,9 \%$ und in der Gruppe von Murakami [3] bei 1034 Probanden bei 3,1\%. Lediglich in der Gruppe um Mishriki [4]war bei kleiner Probandenzahl von 292 Patienten die
Tumorfindungsrate mit 5,4\% höher. Auf dem letzten AUA-Kongress 2016 in San Diego stellten Samson et al. eine Studie vor, in der bei 1761 Patienten lediglich 14 Malignome gefunden wurden, also bei 0,85\% der Patienten.

In der vorliegenden Studie wurden in einem gynäkologischen Tertiary-Care-Setting postmenopausale Frauen mit asymptomatischer Mikrohämaturie untersucht, die keinen Anhalt für eine gynäkologische Blutungsursache hatten. Im Hinblick auf Risikofaktoren hat diese Gruppe orientiert am Lebensalter ein höheres, im Hinblick auf das Geschlecht ein geringeres Risiko für ein urologisches Malignom, insbesondere für ein Urothelkarzinom. Die Diagnostik wurde entsprechend der AUALeitlinie mit Zystoskopie und CT durchgeführt. Bei 237 Frauen fanden sich in 1,3\% der Fälle Malignome, und zwar 2 Blasenkarzinome und 1 Nierenzellkarzinom. Diese Zahl liegt im Rahmen dessen, was wir aus bisherigen Studien kennen.

Es gibt weltweit wenige Leitlinien zur Mikrohämaturieabklärung ( $\mathbf{O}$ Tab.1). Die spezifischste für die Urologie ist die AUALeitlinie, die 2012 revidiert und deutlich verschärft wurde: Nach nur einmaliger Sicherung einer Mikrohämaturie (>3 Erythrozyten/Gesichtsfeld) werden dort Zystoskopie und multiphasisches CT-Urogramm vorgegeben. Die Sonografie wird nur als Ersatzuntersuchung genannt, die Urinzytologie abgelehnt. Andere Leitlinien empfehlen 2-3 Urinuntersuchungen, teils als Sediment, teils als Teststreifenunteruntersuchung, vor Beginn der weiteren Diagnostik. Alle fordern die Anamnese auf Risikofaktoren und beschreiben die untere Altersgrenze für die invasive Diagnostik

Tab. 1 Die Diagnostik der Mikrohämaturie in Leitlinien.

\begin{tabular}{|c|c|c|c|c|}
\hline & AUA [5] 2012 & $\begin{array}{l}\text { Kanada [6] } \\
2009\end{array}$ & UK [7] 2008 & $\begin{array}{l}\text { DE: (DEGAM) } \\
\text { [8] } 2013\end{array}$ \\
\hline Testmethode & Urinsediment & Urinsediment & Teststreifen & Teststreifen \\
\hline Grenzwert & $>3 / G F$ & $>2$ & $>1+$ & 10 Ery/ul \\
\hline Zahl der Tests & 1 & 2 & $2-3$ & $2-3$ \\
\hline $\begin{array}{l}\text { Diagnostik, } \\
\text { primär }\end{array}$ & $\begin{array}{l}\text { Nephrologie?* } \\
\text { KEINE Urinzyto- } \\
\text { logie/ Biomar- } \\
\text { ker. } \\
\text { Zystoskopie und } \\
\text { CT, alt. MRT, US }\end{array}$ & $\begin{array}{l}\text { Nephrologie?* } \\
\text { Sonografie } \\
\text { Urinzytologie }\end{array}$ & Nephrologie?* & Nephrologie?* \\
\hline $\begin{array}{l}\text { Diagnostik, } \\
\text { sekundär }\end{array}$ & & $\begin{array}{l}\text { Zystoskopie } \\
\text { CT }\end{array}$ & $\begin{array}{l}\text { Überweisung } \\
\text { - Nephrologie } \\
\text { - Urologie }\end{array}$ & $\begin{array}{l}\text { Überweisung } \\
\text { - Nephrologie } \\
\text { - Urologie }\end{array}$ \\
\hline $\begin{array}{l}\text { Risikofaktoren } \\
\text { (OBox) }\end{array}$ & beachten & beachten & beachten & beachten \\
\hline Altersgrenze & 35 Jahre & 40 Jahre & 40 Jahre & 40 Jahre \\
\hline
\end{tabular}

mit 35 (AUA) oder 40 Jahren. Die deutsche Leitlinie der DEGAM bezieht ausdrücklich die Medizinische Fachangestellte (MFA, „Arzthelferin“) in den Abklärungsprozess ein.

\section{Herausforderung für Patienten}

Unabhängig von der Kostenfrage ist die Mikrohämaturieabklärung eine Herausforderung für den Patienten, denn sie schließt Patientenbelastung durch Zystoskopie, Strahlenbelastung und Kontrastmittelrisiken ein. Und wir sollten die psychische Belastung nicht vergessen, weil der Begriff „Blut“ vom Patienten nahezu automatisch mit „Krebs“ assoziiert wird. Und das obwohl wir aus der Literatur wissen, dass bei der Mikrohämaturieabklärung allenfalls in 5\% unselektierter Patienten - vermutlich deutlich seltener ein Tumor zu finden ist. Darüber hinaus ist bedenkenswert, dass keine der Studien eine Kontrollgruppe mit normalem Urinbefund hatte, und wir wissen, dass bei jeder Abklärung des Harntrakts - gleichgültig aus welchem Anlass - Tumoren entdeckt werden können. Dies dürfte die Zahlen unserer Fragestellung weiter relativieren. Vergleichen wir unsere Zahlen mit denen der PSA-gestützten Prostatakarzinomdiagnostik, so finden wir dort eine 10 fach höhere Rate damit entdeckter Tumoren, und dennoch wurden wir von Epidemiologen und der Presse heftig gescholten wegen eines Missverhältnisses von Untersuchungsergebnissen und $\mathrm{Pa}-$ tientenbelastung.

\section{Was tun?}

Insgesamt müssen wir unser „Bauchgefühl" korrigieren und fühlen uns in einem Dilemma, gerade, wenn wir Kliniker sind (nur 2-3\% der Patienten eines Quartals werden stationär eingewiesen und nur die schwerer Erkrankten): Bei der Mikrohämaturieabklärung finden wir vergleichsweise selten Tumoren im Harntrakt bei doch deutlicher Patientenbelastung durch die Diagnostik, und vieles spricht gegen ein pauschalisiertes und striktes Vorgehen, so wie es die AUA-Leitlinie vorgibt. So ist eine daran angelehnte Stufendiagnostik denkbar, bei der zunächst eine nicht belastende Urindiagnostik mit mehrfachem Urinsediment, Erythrozytenmorphologie, Infektausschluss, Proteinuriediagnostik und Urinzytologie durchgeführt wird, gefolgt von der Sonografie, die in Deutschland in der Hand des Urologen ist. Ein wesentlicher Punkt ist es, Risikofaktoren für ein Malignom im Harntrakt ( $\odot$ Box) zu erfassen. Auf dieser Basis kann dann 


\section{Risikofaktoren für Malignome} im Harntrakt

- Männliches Geschlecht

- Alter: >35-40 Jahre

- (Ex-)Raucher

- Chemikalienexposition

- Phenacetinabusus

- Cyclophosphamid-Therapie

- Miktionsbeschwerden

- Bestrahlung im Becken

differenziert die weitere Diagnostik mit Zystoskopie, Schnittbilduntersuchungen und ggf. weiteren Untersuchungen mit dem Patienten abgesprochen werden, wenn er nicht vor lauter Angst (MFA: „Sie haben aber Bluhut im Urin. Das kann Krebs sein!“) allem zustimmt.

\section{Prof. Dr. Helmut Haas, Heppenheim}

Literatur

1 Jung H, Gleason JM, Loo RK et al. Association of hematuria on microscopic urinalysis and risk of urinary tract cancer. J Urol 2011; 185 : 1698-1703

2 Loo RK, Lieberman SF, Slezak JM. Stratifying risk of urinary tract malignant tumors in patients with asymptomatic microscopic hematuria. Mayo Clin Proc 2013; 88: 129-318

3 Murakami S, Igarashi T, Hara S, Shimazaki J. Strategies for asymptomatic microscopic hematuria: a prospective study of 1,034 patients.J Urol 1990; 144: 99-101

4 Mishriki SF, Nabi G, Cohen NP. Diagnosis of urologic malignancies in patients with asymptomatic dipstick hematuria: prospective study with 13 years' follow-up. Urology 2008; 71: 13-16

5 Davis R, Jones JS, Barocas DA et al. Diagnosis, Evaluation and Follow-Up of Asymptomatic Microhematuria (AMH) In Adults: AUA Guideline. J Urol, 2012; 188 (Suppl.): 2473

6 Wollin T, Laroche B, Psooy K. Canadian guidelines for the management of asymptomatic microscopic hematuria in adults. Can Urol Assoc J 2009; 3: 77-80

7 Anderson J, Fawsett D, Feehally J et al. Joint Consensus Statement on the Initial Assessment of Haematuria. Im Internet: http:// www.renal.org/docs/default-source/whatwe-do/RA-BAUS_Haematuria_Consensus_ Guidelines.pdf?sfvrsn $=0$

8 Mainz A. Nicht-sichtbare Hämaturie. AWMF-Registernr. 053/028. Klasse S1. Im Internet: http://www.awmf.org/uploads/ tx_szleitlinien/053-028-_S10_Nicht_sichtbare_Hämaturie_2013-10.pdf 